\title{
Association between goal-striving stress and rapid kidney function decline among African Americans: the Jackson Heart Study
}

\author{
Loretta Cain-Shields (D) , ${ }^{1}$ LáShauntá Glover, ${ }^{2}$ Bessie Young, ${ }^{3,4}$ Mario Sims ${ }^{5}$
}

\begin{abstract}
Department of Data
Science, John D. Bower

School of Population Health,

University of Mississippi

Medical Center, Jackson,

Mississippi, USA

${ }^{2}$ Department of

Epidemiology, University of North Carolina at Chapel Hill Gillings School of Global Public Health, Chapel Hill,

North Carolina, USA

${ }^{3}$ Division of Nephrology and

Kidney Research Institute, University of Washington, Seattle, Washington, USA ${ }^{4}$ Veterans Affairs, Hospital and Specialty Medicine and Center for Innovation, Seattle, Washington, USA ${ }^{5}$ School of Medicine, Jackson Heart Study, University of Mississippi Medical Center Jackson, Mississippi, USA
\end{abstract}

\section{Correspondence to} Dr Loretta Cain-Shields, Department of Data Science, Population Health, University of Mississippi Medical Center, Jackson, MS 39216, USA; Icain@umc.edu

Accepted 6 October 2020 Published Online First 17 December 2020 John D Bower School of

\section{ABSTRACT}

African Americans (AAs) are disproportionately affected by kidney disease and also report higher psychosocial stressors than other racial groups. Goalstriving stress (GSS) is an understudied psychosocial stressor related to attempting to accomplish one's life goals. Given the numerous social determinants that contribute to health inequities among AAs, stress from goal striving may also disproportionately affect the health of AAs and in particular kidney disease outcomes. The objective of this study was to explore the association between GSS and rapid kidney function decline (RKFD) in an $A A$ cohort. Using examination 1 (2000-2004) and examination 3 (2009-2013) data from the Jackson Heart Study ( $n=2630)$, we examined associations of baseline levels of GSS with RKFD among AAs using multivariable Poisson regression models, adjusting for sociodemographics, health behaviors, chronic disease and discrimination. We also explored baseline cortisol as a mediator. The incidence of RKFD in this sample was $7.34 \%$ (mean years of follow-up: $8.06 \pm 0.84$ years). The mean GSS score was $3.80( \pm 4.88)$ and total GSS score ranged from 0 to 36. Those who reported high (vs low) GSS were 1.60 times more likely to experience RKFD after full adjustment (incidence rate ratio (IRR) 1.60; $95 \% \mathrm{Cl}$ 1.11 to $2.14, p=0.01$ ). After confirming cortisol as a mediator and adding it to the model, those who reported high (vs low) GSS had 1.58 times the rate of RKFD (IRR 1.58; $95 \% \mathrm{Cl} 1.09$ to $2.30, p=0.0153$ ). Stress related to not achieving goals was associated with a greater risk of RKFD in this sample of AAs.

\section{SLinked}

- http://dx.doi.org/10. 1136/jim-2021-001787

Check for updates

(C) American Federation for Medical Research 2021. No commercial re-use. See rights and permissions. Published by BMJ.

To cite: Cain-Shields $L$, Glover LS, Young B,

et al. J Investig Med

2021;69:382-387.

\section{INTRODUCTION}

Kidney disease is a significant public health problem. The overall chronic kidney disease (CKD) prevalence in the US population is about $15 \%$ or (30 million people), and adults with diabetes and high blood pressure are at higher risk of developing CKD. ${ }^{1}$ Complications of CKD include end-stage renal disease (ESRD), heart disease, stroke, anemia, infections, low calcium levels, high phosphorus levels, high potassium levels, loss of appetite, excess fluids, depression and higher risk of mortality. ${ }^{1}$ The most recent estimate for Medicare expenditures for beneficiaries with CKD exceeded $\$ 84$ billion

\section{Significance of this study}

What is already known about this subject?

- Kidney disease is a public health problem.

- Ethnic and racial disparities in kidney disease are also a public health problem.

- Traditional modifiable risk factors only partly explain racial disparities in chronic kidney disease.

- Stressors disproportionately affect racial and ethnic minorities and are nontraditional risk factors that may lead to kidney disease.

\section{What are the new findings?}

- Goal-striving stress is associated with rapid kidney function decline in African Americans.

- Controlling for baseline cortisol only partially mediated this association.

- There was no effect modification by sociodemographics, health behaviors or chronic disease.

\section{How might these results change the focus} of research or clinical practice?

- Researchers and clinicians should continue to explore non-traditional risk factors in an effort to explain and prevent racial disparities in kidney disease.

in 2017 and for those with ESRD, an additional $\$ 36$ billion, for a total of \$120 billion. ${ }^{2}$

Racial and ethnic disparities in kidney disease are also a significant public health problem. ${ }^{3}$ African Americans (AAs) are 3 times more likely to progress to ESRD, and AAs develop albuminuria at higher rates when compared with their white counterparts. ${ }^{14}$ Studies have found that traditional risk factors (e.g., cardiovascular disease (CVD), hypertension (HTN) and diabetes) only partially explain the higher rates of kidney disease among AAs; therefore, psychosocial factors, such as stressors, should be explored as contributors to the racial disparities found in kidney disease trends. ${ }^{5-7}$

It is known that psychological stressors create demands that require an individual's internal environment to maintain stability in response to the stress (i.e., allostasis) through changes 
in the autonomic nervous, immune, metabolic, cardiovascular, and endocrine systems. ${ }^{89}$ McEwen (1993) suggests that increased allostatic load (AL) occurs in 4 ways: (1) frequent stress, (2) inability to adapt to repeated stressors, (3) inability to shut off stress response, and (4) an unbalanced allostatic response. ${ }^{10}$ Increased AL may predispose individuals to disease, including HTN, CVD, and kidney disease. ${ }^{9}$ The relationship between psychosocial stress with HTN and CVD is well documented. ${ }^{11-14}$ However, research regarding psychosocial stressors and kidney disease is limited. Suggested mechanisms between psychosocial stressors and kidney disease include increased sympathetic nervous system activity, alternations in the hypothalamicpituitary-adrenal axis, and changes in inflammatory cytokines and endothelin-A. 5715

One psychosocial stressor that has not been adequately examined in relation to kidney disease is goal-striving stress (GSS). GSS is the discrepancy between aspiration and achievement, weighted by the level of disappointment experienced if goals are not reached. ${ }^{16}$ GSS captures negative emotions and challenges related to upward mobility and attaining life goals. ${ }^{17}$ Given the racial inequalities of wealth, wages, educational attainment, perceived job control and occupational stress, stress from goal striving may disproportionately affect AAs. ${ }^{19-25}$ Therefore, AAs may be more susceptible to increased AL through McEwen's first mechanism, frequent stress. ${ }^{10}$ Because stress hormones are both metabolized and cleared by the kidneys, those with disproportionate amounts of stress may be more susceptible to kidney disease. ${ }^{7}$

Cross-sectional research has established positive relationships between GSS and prevalent CKD in AAs, and longitudinal research has established positive relationships between GSS and incident CVD in AAs. ${ }^{26}{ }^{27}$ However, the temporal association between GSS and kidney disease has not been explored. One temporal measure of kidney disease is rapid kidney function decline (RKFD), which averages the change in kidney function (i.e., estimated glomerular filtration rate, eGFR) across years. Assessing the association between GSS and RKFD would add temporality and possibly improve our understanding of psychosocial stress and kidney disease in AAs. Therefore, our study examined the associations of baseline GSS with RKFD among AAs in the Jackson Heart Study (JHS). We hypothesize that those who report high (vs low) baseline GSS will have a greater risk for RKFD over time. We also hypothesize that baseline plasma cortisol will partially mediate the association between GSS and RKFD.

\section{METHODS}

The JHS is a large, prospective cohort study of the etiology of CVD in AAs who reside in the tri-county area of the Jackson, Mississippi (MS) metropolitan area (Hinds, Madison, and Rankin counties). Of the 5306 JHS participants, 31\% were recruited from the Atherosclerosis Risk in Communities study, 22\% were family members, $17 \%$ were randomly selected, and 30\% were community volunteers. Participants provided medical history, physical and biochemical measurements, diagnostic procedures, and DNA. ${ }^{28}$ Our study used data from examination 1 (20002004) and examination 3 (2009-2013). At baseline, there were 5306 participants 21-94 years of age enrolled, and at examination 3, 3819 participants (age 28-100 years) remained. Details on the study have been published elsewhere. ${ }^{2829}$ Of the 3819 participants present at examination 3 , we excluded those who had kidney disease at baseline $(\mathrm{n}=468)$, defined as $<60 \mathrm{~mL} / \mathrm{min} / 1.73 \mathrm{~m}^{2}$, a self-reported history of CKD, the use of dialysis, or the presence of albuminuria. We also excluded those with missing baseline GSS values $(n=67)$, missing serum creatinine values at examination $3(n=88)$, and missing covariate information $(n=566)$, leaving a sample of 2630 participants.

\section{Goal-striving stress}

Baseline GSS was defined as the difference between aspiration and achievement, both using a 10-step ladder (1worst possible way of life and 10-best possible way of life), weighted by disappointment experienced if the goal was not achieved by the following year (ranging from very disappointed to not at all disappointed using a 4-point ladder; the GSS score was derived using the following equation: (aspiration-achievement) $\times$ disappointment. $^{16} 17262730$ GSS scores ranged from 0 to 36 . A higher GSS score indicated greater stress from goal striving. GSS was analyzed both continuously and categorically (low (0-1), moderate $(2-4)$, and high $(\geq 5))$ to explore both the continuous and threshold effects of GSS with RKFD.

\section{Rapid kidney function decline}

eGFR was determined using the Chronic Kidney Disease Epidemiology Collaboration (CKD-EPI) equation to measure kidney function based on the validated serum creatinine. ${ }^{31}$ Serum creatinine was measured with a multipoint enzymatic spectrophotometric assay at the baseline visit. ${ }^{32}$ An eGFR $<60 \mathrm{~mL} / \mathrm{min} / 1.73 \mathrm{~m}^{2}$ was indicative of CKD. RKFD was determined based on measurement of eGFR from visit 1 to visit 3, divided by the number of years from visit 1 to visit $3 .^{33} 34$ RKFD was defined as $>4 \mathrm{~mL} / \mathrm{min} / 1.73 \mathrm{~m}^{2} /$ years as described in previous research regarding RKFD. ${ }^{334}$ Currently, there are no official clinical cut-points for RKFD.

Serum creatinine was measured using a multipoint enzymatic spectrophotometric assay at baseline entry into the study using the Vitros Ortho-Clinical Diagnostics Analyzer (Raritan, New Jersey). ${ }^{35}$ Serum creatinine was remeasured in 2006 for 206 participants using the enzymatic method on a Roche chemistry analyzer (Roche Diagnostics, Indianapolis, Indiana). In order to harmonize serum creatinine measurements across study examinations, we calibrated all examination 1 serum creatinine measurements to those at examination 3, using the isotope dilution mass spectrometry (IDMS)-traceable method. ${ }^{32}$ Because the CKD-EPI equation, used to determine kidney disease, is designed to be used with IDMS-calibrated serum creatinine values only, $^{31}$ serum creatinine measurements at examination 1 were optimally calibrated using a Deming regression model, as described previously. ${ }^{32}$

\section{Covariates}

Demographic, health behavior, risk factor and discrimination covariates were chosen based on their known relationships with kidney disease and stress. Baseline covariates included age (continuous), sex (men (referent)/women), 
education (less than high school graduate (referent), high school graduate, attended college), smoking status (current smoker, quit $<12$ months ago, and never smoked or quit $\geq$ monthsago (referent)), alcohol use within the last year (yes/no (referent)), and physical activity based on the American Heart Association's Life's Simple Seven criteria poor (0 minute of moderate physical activity/week; and 0 minute of vigorous physical activity/week), intermediate health $(0<$ minutes of moderate physical activity/week $<150$ or $0<$ minutes of vigorous physical activity/week $<75$ or 0 $<$ minutes of combined moderate and vigorous physical activity/week <150) (referent).

Other baseline covariates included were body mass index (BMI) categories (obese, overweight, normal (referent)), HTN status (normal (referent): $0<$ systolic blood pressure $<120$ and $0<$ diastolic blood pressure $<80$, pre-HTN: $120 \leq$ systolic blood pressure $<140$ or $80 \leq$ diastolic blood pressure $<90$, HTN: systolic blood pressure $\geq 140$ or diastolic blood pressure $\geq 90$ ), diabetes status (non-diabetes (referent) fasting plasma glucose (FPG) $<100$ and HbA1c $<5.7$ and no diabetes medication use), pre-diabetic (100 $\leq$ FPG $<126$ or $5.7 \leq$ HbA1c $<6.5$ and no diabetes medication use), diabetic (FPG $\geq 126$ or HbA1c $<6.5$ and diabetes medication use), CVD history (defined as self-reported physician-diagnosed cardiovascular history (CVD/no CVD $($ referent $)))$, and plasma cortisol $(\mu \mathrm{g} / \mathrm{dL})$.

The burden of perceived lifetime discrimination was also included (continuous score range 1-4) as a potential psychosocial confounder in the association between GSS and RKFD, since it is a common stressor reported among AAs. ${ }^{36-38}$ Burden of lifetime discrimination was measured using 3 questions (codes): 'When you had experiences like these over your lifetime, have they been-very stressful (4), moderately stressful (2.5), or not stressful (1)?', 'Overall, how much has discrimination interfered with you having a full and productive life? - lot (4), some (3), a little (2), or not at all (1)?', and 'Overall, how much harder has your life been because of discrimination- lot (4), some (3), a little (2), or not at all (1)?' Responses were averaged to create a mean score of discrimination burden, ranging from 1 to 4 . Higher scores indicated greater burden of discrimination. Internal consistency for this scale was $0.63 .{ }^{39}$

\section{Statistical analysis}

Observations with missing covariates were compared with the analytical sample on age, sex, educational attainment, alcohol use, smoking status, HTN status, diabetes status, and CVD history and discrimination score. Missing observations were statistically different from the analytical sample by mean age ( 57.37 vs 53.28 years), college attendance $(51.73 \%$ vs $71.25 \%)$, current alcohol use $(42.10 \%$ vs $49.6 \%)$, current smoking ( $15.53 \%$ vs $11.06 \%)$, HTN (24.97\% vs $17.19 \%)$, diabetes ( $27.08 \%$ vs 17.53$)$ and CVD history $(15.28 \%$ vs $6.20 \%)$, respectively. Therefore, those who were missing from this sample were older, less likely to attend college and use alcohol, more likely to smoke and more likely to have HTN, diabetes, and a history of CVD.

Unadjusted differences in baseline characteristics were assessed across tertiles of GSS using $\chi^{2}$ test for categorical variables and Kruskal-Wallis test for non-normal continuous variables. There were no normal continuous variables.
Multivariable Poisson regression analyses estimated the associations of baseline GSS levels and continuous GSS with RKFD, where incidence rate ratios (IRRs, 95\% CIs) estimated the relative rate of RKFD. Model 1 was adjusted for demographics: age, sex, and education. Model 2 was additionally adjusted for health behaviors: smoking status, alcohol use and physical activity. Model 3 was additionally adjusted for risk factors: HTN, BMI, diabetes mellitus, and history of CVD. Model 4 was additionally adjusted for an important psychosocial risk factor: perceived burden of lifetime discrimination. Model 5 additionally adjusted for plasma cortisol $(\mu \mathrm{g} / \mathrm{dL})$ after mediation was confirmed.

Because kidney disease is known to vary by sex and the above-mentioned risk factors, interaction tests for GSS with sex, HTN, BMI, diabetes mellitus, and history of CVD were conducted, and effect modification was explored. To test the theory that stress causes a disproportionate amount of stress to be filtered through kidneys, causing kidney disease, a mediation analysis was performed using the Baron and Kenny method, ${ }^{40}$ testing baseline plasma cortisol $(\mu \mathrm{g} / \mathrm{dL})$ as the mediator. To conclude mediation, Baron and Kenny proposed the following: (1) the dependent and independent variables are correlated, (2) the independent and mediator variables are correlated, and (3) controlling for the mediator attenuates the relationship between the independent and dependent variables. ${ }^{40}$ All analyses were performed using SAS 9.3 (SAS Institute). Statistical significance was inferred at two-sided $\mathrm{p}<0.05$.

\section{RESULTS}

RKFD occurred in 7.34\% ( $\mathrm{n}=193)$ of the sample population (table 1). At baseline, participants who reported high (vs low) GSS had higher eGFR at visit $1(102.96 \pm 18.22$ vs $93.35 \pm 16.61)$, higher eGFR at visit $3(93.20 \pm 21.97$ vs $84.17 \pm 19.89)$, higher GSS score $(9.96 \pm 5.55$ vs $0.25 \pm 0.43)$, were more likely to be younger $(48.21 \pm 11.23$ vs $58.04 \pm 10.58)$, female $(66.90 \%$ vs $62.61 \%)$, college attendees $(72.28 \%$ vs $66.91 \%)$, current smokers $(15.70 \%$ vs $8.48 \%)$, use alcohol $(54.88 \%$ vs $44.94 \%)$, obese $(55.87 \%$ vs $54.65 \%)$, and have a higher discrimination score (2.49 vs $2.26)$. They were less likely to have poor physical activity (40.59\% vs $48.21 \%)$, hypertension ( $13.86 \%$ vs $20.33 \%)$, diabetes $(15.56 \%$ vs $20.53 \%)$, and less likely to have a history of CVD (4.53\% vs $8.38 \%$ ) (table 1$)$.

The mean follow-up time from visit 1 to visit 3 was $8.06 \pm 0.84$ years. After adjustment for age, sex and education, participants who reported high (vs low) GSS had 1.73 times the rate of RKFD (IRR 1.73; 95\% CI 1.12 to 2.48, $\mathrm{p}=0.0031$ ) (table 2). This association slightly attenuated across models 2,3 and 4 , but remained significant $(p=0.01)$ (table 2). Continuous GSS was significantly associated with RKFD in models 1 and 2 but not in models 3 and 4 .

The interactions between GSS with sex, HTN, BMI, diabetes mellitus, and history of CVD were not significant. In addition, there was no effect modification by these variables. In the mediation analysis, controlling for all covariates, GSS was significantly associated with RKFD $(p=0.0134)$, plasma cortisol $(p=0.0476)$, and RKFD, when controlling for plasma cortisol $(p=0.0173)$. The result of adding cortisol to the model is shown as model 5 in table 2. Adding plasma cortisol to the model resulted in a $2 \%$ 
Table 1 Sample population demographics by goal-striving stress (GSS) levels, JHS (2000-2004)

\begin{tabular}{|c|c|c|c|c|c|}
\hline Variable & $\begin{array}{l}\text { Entire cohort } \\
(n=2630)\end{array}$ & $\begin{array}{l}\text { Low GSS } \\
(n=979)\end{array}$ & $\begin{array}{l}\text { Moderate GSS } \\
(n=944)\end{array}$ & $\begin{array}{l}\text { High GSS } \\
(n=707)\end{array}$ & $P$ value \\
\hline RKFD (\%) & $193(7.34)$ & $70(7.15)$ & $61(6.46)$ & $62(8.77)$ & 0.1972 \\
\hline $\mathrm{eGFR}_{\text {visit1 }}($ mean $\mathrm{SD})$ & $98.25 \pm 17.40$ & $93.35 \pm 16.61$ & $98.76 \pm 16.63$ & $102.96 \pm 18.22$ & $<0.001$ * \\
\hline 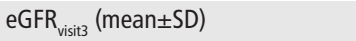 & $88.40 \pm 21.06$ & $84.17 \pm 19.89$ & $89.19 \pm 20.70$ & $93.20 \pm 21.97$ & $<0.001$ * \\
\hline $\mathrm{eGFR}_{\text {visit3 }}-\mathrm{eGFR}_{\text {visit1 }}($ mean $\pm \mathrm{SD})$ & $-9.85 \pm 15.32$ & $-10.17 \pm 15.11$ & $-9.57 \pm 14.61$ & $-9.76 \pm 16.48$ & 0.6369 \\
\hline GSS $($ mean \pm SD) & $3.80 \pm 4.88$ & $0.25 \pm 0.43$ & $2.89 \pm 0.89$ & $9.96 \pm 5.55$ & $<0.001^{*}$ \\
\hline Age $($ mean $\pm S D)$ & $53.28 \pm 11.53$ & $58.04 \pm 10.58$ & $52.15 \pm 10.79$ & $48.21 \pm 11.23$ & $<0.001^{*}$ \\
\hline Females (\%) & $1646(62.59)$ & $613(62.61)$ & $560(59.32)$ & $473(66.90)$ & $0.007^{*}$ \\
\hline Attended college (\%) & $1874(71.25)$ & $655(66.91)$ & $708(75.00)$ & $511(72.28)$ & $<0.001$ * \\
\hline Current smoker (\%) & $291(11.06)$ & $83(8.48)$ & $97(10.28)$ & $111(15.70)$ & $<0.001^{*}$ \\
\hline Use alcohol (\%) & $1305(49.62)$ & $440(44.94)$ & $477(50.53)$ & $388(54.88)$ & $0.002^{*}$ \\
\hline Poor physical activity health (\%) & $1152(43.80)$ & $472(48.21)$ & $393(41.63)$ & $287(40.59)$ & $0.002^{*}$ \\
\hline Obese $(\%)$ & $1422(54.07)$ & $535(54.65)$ & $492(52.12)$ & $395(55.87)$ & 0.1419 \\
\hline Hypertension (\%) & $452(17.19)$ & $199(20.33)$ & $155(16.42)$ & $98(13.86)$ & $<0.001^{*}$ \\
\hline Diabetes $(\%)$ & $461(17.53)$ & $201(20.53)$ & $150(15.89)$ & $110(15.56)$ & $<0.001^{*}$ \\
\hline Cardiovascular disease history (\%) & $163(6.20)$ & $82(8.38)$ & $42(4.45)$ & $32(4.53)$ & $0.001^{*}$ \\
\hline Discrimination (1-4) $($ mean \pm SD) & $2.35 \pm 0.76$ & $2.26 \pm 0.79$ & $2.33 \pm 0.74$ & $2.49 \pm 0.74$ & $<0.001$ * \\
\hline
\end{tabular}

$P$ values were calculated using $\chi^{2}$ and Kruskal-Wallis tests as appropriate.

* Significant $p$ values.

eGFR, estimated glomerular filtration rate; JHS, Jackson Heart Study; RKFD, rapid kidney function decline.

decrease in the relative rate of RKFD when compared with the previous model, indicating only partial mediation. In model 5, participants who reported high (vs low) GSS had 1.58 times the rate of RKFD (IRR $1.58 ; 95 \%$ CI 1.09 to $2.30, \mathrm{p}=0.0153)$.

\section{DISCUSSION}

In our sample, GSS was associated with RKFD after adjusting for demographics, health behaviors, risk factors, and burden of discrimination. Psychological stress increases $\mathrm{AL}$ in the autonomic nervous, immune, metabolic, cardiovascular, and endocrine systems in an effort to maintain physiological stability. ${ }^{89}$ These extra physiological demands may cause internal systems to break down, predisposing individuals to chronic disease, including kidney disease. ${ }^{9}$ Because stress hormones are both metabolized and cleared by the kidneys, those with greater amounts of stress may be more susceptible to kidney disease. ${ }^{7}$ However, after testing for the mediating effects of cortisol, a common stress hormone, the relative rate was only attenuated by $2 \%$, indicating partial mediation. Therefore, other stress hormones should be explored in an effort to explain the association between GSS and RKFD.

Previous work has found positive associations between GSS and outcomes related to RKFD. Sellers et al found that as GSS increased, prevalent HTN, a kidney disease risk factor, increased in 3570 AAs, 891 Whites, and 1621 Caribbean descent participants from the National Survey of American Life (NSAL). ${ }^{41}$ In contrast, we found a higher prevalence of HTN in the lowest category of GSS as shown in our demographics table. There are several explanations for the difference in findings between GSS and HTN among the 2 studies. One, our population demographics are different from that of the NSAL study. Also, in the NSAL study, HTN was self-reported. Last, our look at HTN and GSS was only descriptive and unadjusted for potential confounders, as this was not our outcome variable of interest. Glover et $a l^{27}$ found a significant positive association between GSS and incident CVD, a kidney disease risk factor, among AAs in the JHS $(n=4648)$. Cain $e$ t $a l^{26}$ found a significant positive association between GSS and prevalent CKD among the AA participants of the JHS (n=4967). Like the previously mentioned studies, the current study found a significant association between GSS and increased risk of RKFD.

This study reports the association between an understudied psychosocial stressor that is particularly relevant to AAs and kidney function decline in a large prospective study of AAs, which adds to both the kidney disease and GSS literature. GSS has not been previously linked to incident CKD in any population, especially a population that may be more susceptible to stress and kidney disease. In addition, the associations reported adjusted for multiple risk factors including demographics, health behaviors, chronic disease and discrimination which may impact the relationship between GSS and RKFD. Despite these strengths, this study also has limitations. Although we used an AA sample, the findings may only be generalized to AAs in the Jackson, MS area. Additionally, there may have been other factors that could explain the association between GSS and RKFD that were not identified, such as family history or genetic susceptibility to kidney disease. Also, using a completecase analysis approach may not accurately represent the original population from which the sample was taken. It is also important to note that there is currently no clinical cutpoint for RKFD and that eGFR decline was not evaluated as a continuous variable.

GSS was associated with a greater risk of RKFD among AAs in the JHS. Minorities typically report greater stressors and have lower socioeconomic status than their white counterparts, ${ }^{42}$ which influences health disparities. It is well known that stress increases the risk of disease and 
Table 2 The association between baseline goal-striving stress (GSS) and rapid kidney function decline (incidence rate ratio (IRR) $95 \% \mathrm{Cl})$, JHS 2000-2013 ( $\mathrm{n}=2630)$

\begin{tabular}{|c|c|}
\hline & Statistic \\
\hline Variable & IRR (95\% Cl) \\
\hline \multicolumn{2}{|l|}{ Model 1} \\
\hline Low GSS & Reference \\
\hline Moderate GSS & $1.15(0.81$ to 1.63$) p=0.4374$ \\
\hline High GSS & 1.73 (1.120 to 2.48$) p=0.0031$ * \\
\hline Continuous GSS & $\begin{array}{l}0.032(0.006 \text { to } 0.059) \\
p=0.0152^{*}\end{array}$ \\
\hline \multicolumn{2}{|l|}{ Model 2} \\
\hline Low GSS & Reference \\
\hline Moderate GSS & 1.13 (0.80 to 1.61$) p=0.4758$ \\
\hline High GSS & 1.63 (1.13 to 2.34$) \mathrm{p}=0.0085^{*}$ \\
\hline Continuous GSS & $\begin{array}{l}0.028(-0.001 \text { to } 0.053) \\
p=0.0396^{*}\end{array}$ \\
\hline \multicolumn{2}{|l|}{ Model 3} \\
\hline Low GSS & Reference \\
\hline Moderate GSS & $1.18(0.83$ to 1.67$) p=0.3669$ \\
\hline High GSS & $1.61(1.12$ to 2.32$) p=0.0101^{*}$ \\
\hline Continuous GSS & $\begin{array}{l}0.026(-0.001 \text { to } 0.052) \\
p=0.0557\end{array}$ \\
\hline \multicolumn{2}{|l|}{ Model 4} \\
\hline Low GSS & Reference \\
\hline Moderate GSS & $1.17(0.83$ to 1.67$) p=0.3707$ \\
\hline High GSS & 1.60 (1.11 to 2.14$) p=0.0119$ \\
\hline Continuous GSS & $\begin{array}{l}0.025(-0.002 \text { to } 0.051) \\
p=0.0665\end{array}$ \\
\hline \multicolumn{2}{|l|}{ Model 5} \\
\hline Low GSS & Reference \\
\hline Moderate GSS & $1.16(0.82$ to 1.65$) p=0.4072$ \\
\hline High GSS & $1.58(1.09$ to 2.30$) p=0.0153$ \\
\hline
\end{tabular}

Model 1: adjusted for age, sex, and education.

Model 2: model 1+smoking status, alcohol use status, and physical activity status.

Model 3: model 2+body mass index (BMI), hypertension, diabetes, and cardiovascular disease.

Model 4: model 3+discrimination.

Model 5: model 4+baseline cortisol.

*Significant $p$ values.

JHS, Jackson Heart Study.

that traditional modifiable risk factors only partly explain racial disparities in CKD health. ${ }^{56}$ However, the associations between psychosocial stressors and kidney disease are less known. Future research should consider examining stress related to goal striving with other chronic diseases, such as diabetes and HTN among AAs, as this type of stress may explain racial health disparities in these and other morbidities.

Acknowledgements We wish to thank all the participants and staff of the Jackson Heart Study for their time, effort, and dedication towards this study.

Contributors All authors (LCS, LG, BY and MS) have each substantially contributed to the design of the work and interpretation of data for the work, drafted or revised the work critically for important intellectual content, approved the final version to be published and agreed to be accountable for all aspects of the work in ensuring that questions related to the accuracy or integrity of any part of the work are appropriately investigated and resolved. Additionally, LCS acquired and analyzed the data for the work.
Funding The Jackson Heart Study (JHS) is supported and conducted in collaboration with Jackson State University (HHSN268201800013I), Tougaloo College (HHSN268201800014I), the Mississippi State Department of Health (HHSN268201800015I) and the University of Mississippi Medical Center (HHSN268201800010I, HHSN2682018000111, and HHSN268201800012I) contracts from the National Heart, Lung, and Blood Institute (NHLBI) and the National Institute for Minority Health and Health Disparities (NIMHD). MS is supported by grants P60MD002249 and U54MD008176 from NIMHD; 15SFDRN26140001 and P50HL120163 from the American Heart Association. This study was also supported by the National Institute of Diabetes and Digestive and Kidney Diseases grant 1R01DK102134-01 of the National Institutes of Health (NIH) for BY. BY is also supported, in part, by funding from the Veterans Affairs Puget Sound Health Care System. LG is supported by the Genetic Epidemiology of Heart, Lung, and Blood Traits Training Grant (GENHLB) T32 HL129982.

Disclaimer The Veterans Affairs does not endorse any of the statements or opinions advocated by this manuscript. The views expressed in this manuscript are those of the authors and do not necessarily represent the views of the National Heart, Lung, and Blood Institute; the National Institutes of Health; or the US Department of Health and Human Services. The funders had no role in the study design, collection, analysis, interpretation of data, writing the report, or the decision to submit the report for publication.

\section{Competing interests None declared.}

Patient consent for publication Not required.

Ethics approval Written informed consent was provided by all participants and participating institutions' institutional review boards approved the study: Tougaloo College (Tougaloo, MS), Jackson State University (Jackson, MS), and the University of Mississippi Medical Center (Jackson, MS).

Provenance and peer review Not commissioned; externally peer reviewed. Data availability statement No data are available.

\section{ORCID iD}

Loretta Cain-Shields http://orcid.org/0000-0003-2255-8067

\section{REFERENCES}

1 Centers for Disease Control and Prevention. National chronic disease fact sheet, 2017. Available: https://www.cdc.gov/diabetes/pubs/pdf/kidney factsheet.pdf\#: :text=National\%20Chronic\%20Kidney\%20Disease $\% 20$ Fact\% 20Sheet $\% 2$ C $\% 202017 \% 20$ Chronic,Among \%20Adults\%20in\%20the\% 20United\%20States $\% 20$ Fast $\% 20$ Stats

2 United States Renal Data System. US renal data system 2019 annual data report: epidemiology of kidney disease in the United States, 2019. Available: https://www.usrds.org/media/2371/2019-executive-summary.pdf [Accessed Oct 2020].

3 Garcia-Garcia G, Jha V. World kidney day Steering Committee. Chronic kidney disease in disadvantaged populations. Nephrology 2015;20:113-6.

4 Choi Al, Karter AJ, Liu JY, et al. Ethnic differences in the development of albuminuria: the distance study. Am J Manag Care 2011;17:737-45.

5 Bruce MA, Beech BM, Sims M, et al. Social environmental stressors, psychological factors, and kidney disease. J Investig Med 2009;57:583-9.

6 Norton JM, Moxey-Mims MM, Eggers PW, et al. Social determinants of racial disparities in CKD. J Am Soc Nephrol 2016;27:2576-95.

7 Bruce MA, Griffith DM, Thorpe RJ. Stress and the kidney. Adv Chronic Kidney Dis 2015;22:46-53.

8 Sterling P, Eyer J. Allostasis: A new paradigm to explain arousal pathology. In Fisher S, Reason J, eds. Handbook of life stress, cognition and health. New York: John Wiley \& Sons, 1988: 629-49.

9 McEwen BS, Stellar E. Stress and the individual. mechanisms leading to disease. Arch Intern Med 1993;153:2093-101.

10 McEwen BS. Protective and damaging effects of stress mediators. N Eng/ J Med 1998:338:171-9.

11 Sternberg EM, Chrousos GP, Wilder RL, et al. The stress response and the regulation of inflammatory disease. Ann Intern Med 1992;117:854-66.

12 Theorell T, Karasek RA. Current issues relating to psychosocial job strain and cardiovascular disease research [published correction appears in J Occup Health Psychol 1998 Oct;3(4):369]. J Occup Health Psychol 1996;1:9-26.

13 Sims M, Glover LSM, Gebreab SY, et al. Cumulative psychosocial factors are associated with cardiovascular disease risk factors and management among African Americans in the Jackson heart study. BMC Public Health 2020;20:566.

14 Spruill TM, Butler MJ, Thomas SJ, et al. Association between high perceived stress over time and incident hypertension in black adults: findings from the Jackson heart study. J Am Heart Assoc 2019;8:e012139. 
15 Cukor D, Cohen SD, Peterson RA, et al. Psychosocial aspects of chronic disease: ESRD as a paradigmatic illness. J Am Soc Nephrol 2007;18:3042-55

16 Neighbors HW, Sellers SL, Zhang R, et al. Goal-Striving stress and racial differences in mental health. Race Soc Prob/ 2011;3:51-62.

17 Sellers SL, Neighbors HW, Bonham VL. Goal-striving stress and the mental health of college-educated black American men: the protective effects of system-blame. Am J Orthopsychiatry 2011;81:507-18.

18 Seymour P, Kleiner R. Mental illness in the urban Negro community. New York: Free Press, 1966

19 Assari S, Bazargan M. Unequal associations between educational attainment and occupational stress across racial and ethnic groups. Int J Environ Res Public Health 2019;16:3539.

20 Allen AM, Thomas MD, Michaels EK, et al. Racial discrimination, educational attainment, and biological dysregulation among midlife African American women. Psychoneuroendocrinology 2019;99:225-35.

21 Hernandez M, Avery DR, Volpone SD, et al. Bargaining while black: the role of race in salary negotiations. J App/ Psychol 2019;104:581-92.

22 Smith D, Jacobson C. Differences in salaries of physician assistants in the USA by race, ethnicity and sex.J Health Serv Res Policy 2018;23:44-8.

23 Meyer JD. Race-based job discrimination, disparities in job control, and their joint effects on health. Am J Ind Med 2014;57:587-95.

24 Choudhury S. Racial and ethnic differences in wealth and asset choices. Soc Secur Bull 2001;64:1-15.

25 Fuller-Rowell TE, Curtis DS, Doan SN, et al. Racial disparities in the health benefits of educational attainment: a study of inflammatory trajectories among African American and white adults. Psychosom Med 2015;77:33-40.

26 Cain LR, Glover L, Young B, et al. Goal-Striving stress is associated with chronic kidney disease among participants in the Jackson Heart Study. J Racial Ethn Health Disparities 2019;6:64-9.

27 Glover LM, Cain-Shields LR, Spruill TM, et al. Goal-Striving stress and incident cardiovascular disease in blacks: the Jackson heart study. J Am Heart Assoc 2020;9:e015707.

28 Taylor HA, Wilson JG, Jones DW, et al. Toward resolution of cardiovascular health disparities in African Americans: design and methods of the Jackson heart study. Ethn Dis 2005;15:S6-17.

29 Fuqua SR, Wyatt SB, Andrew ME, et al. Recruiting African-American research participation in the Jackson heart study: methods, response rates, and sample description. Ethn Dis 2005;15:S6-29.
30 Cain-Shields LR, Johnson DA, Glover L, et al. The association of goal-striving stress with sleep duration and sleep quality among African Americans in the Jackson Heart Study. Sleep Health 2020;6:117-23.

31 Levey AS, Stevens LA, Schmid CH, et al. A new equation to estimate glomerular filtration rate. Ann Intern Med 2009;150:604-12.

32 Wang W, Young BA, Fülöp T, et al. Effects of serum creatinine calibration on estimated renal function in African Americans: the Jackson heart study. Am J Med Sci 2015;349:379-84.

33 Al-Aly Z, Zeringue A, Fu J, et al. Rate of kidney function decline associates with mortality. J Am Soc Nephrol 2010;21:1961-9.

34 Vigil A, Condés E, Camacho $\mathrm{R}$, et al. Predictors of a rapid decline of renal function in patients with chronic kidney disease referred to a nephrology outpatient clinic: a longitudinal study. Advances in Nephrology 2015;2015:1-8.

35 Carpenter MA, Crow R, Steffes M, et al. Laboratory, reading center, and coordinating center data management methods in the Jackson heart study. Am J Med Sci 2004;328:131-44.

36 Mendez DD, Hogan VK, Culhane J. Institutional racism and pregnancy health: using home Mortgage disclosure act data to develop an index for Mortgage discrimination at the community level. Public Health Rep 2011;126 Suppl 3:102-14.

37 Isaacs J, Sawhil I, Haskins R. Getting ahead or losing ground: economic mobility in America. Brookings institution, 2008. Available: https://www. brookings.edu/wp-content/uploads/2016/06/02_economic_mobility_sawhill. pdf

38 Sims M, Diez-Roux AV, Dudley A, et al. Perceived discrimination and hypertension among African Americans in the Jackson heart study. Am J Public Health 2012;102 Suppl 2:S258-65.

39 Sims M, Wyatt SB, Gutierrez ML, et al. Development and psychometric testing of a multidimensional instrument of perceived discrimination among African Americans in the Jackson heart study. Ethn Dis 2009;19:56-64.

40 Baron RM, Kenny DA. The moderator-mediator variable distinction in social psychological research: conceptual, strategic, and statistical considerations. J Pers Soc Psychol 1986;51:1173-82.

41 Sellers SL, Neighbors HW, Zhang R, et al. The impact of goal-striving stress on physical health of white Americans, African Americans, and Caribbean blacks. Ethn Dis 2012;22:21-8.

42 Williams DR, Jackson JS, et al. Racial differences in physical and mental health: socio-economic status, stress and discrimination. J Health Psychol $1997 ; 2: 335-51$ 